\title{
Thomas Blanke Probleme einer Theorie des Arbeitsrechts
}

Die nachfolgenden Uberlegungen begreifen sich als ein erster Versuch, Fragestellungen zur Interpretation und Analyse arbeitsrechtlicher Denkformen (Ideologien) und Entwicklungen zu entfalten, die in der bisherigen Diskussion selten explizit gestellt worden sind. Dabei soll zunächst in einem kurzen, notwendig globalen Abriß das Dilemma, genauer gesagt: die Unmöglichkeit einer juristischdogmatischen, immanenten Erfassung des Arbeitsrechts aufgezeigt werden (I); daran anschließend werden aus der Kritik neuerer Ansätze zur materialistischen Interpretation arbeitsrechtlicher Ideologien (II) die theoretisch erst noch zu klärenden Voraussetzungen für die Rekonstruktion arbeitsrechtlicher Theoriebildung ansatzweise formuliert (III), die es rechtfertigen, daß wir in diesem Heft drei Artikel über konkrete Streikbewegungen aus jüngster Zeit abdrucken (IV): den Dockarbeiterstreik 1972 in England, den »aktiven Streik « bei LIP in Frankreich und die spontanen Streiks in der BRD 1973.

(I) Arbeitsrecht, d. h. die in rechtliche Form gefaßte institutionelle »Regelung « des Lohnarbeitsverhältnisses, wird durch die bürgerliche Gesellschaft erst hervorgebracht.

Ökonomisch ist es Ausdruck der spezifisch kapitalistischen Produktionsweise, soziologisch Ausdruck des Verhältnisses zwischen den entscheidenden sozialen Klassen. Juristisch: Das Arbeitsrecht ist einerseits bürgerliches Verkehrsrecht (Austausch der Waren Arbeitskraft gegen Geld), andererseits "Sozialrecht" (Sanktionierung der Lebensbedingungen einer sozialen Klasse). Indem es derart unmittelbar auf das Zentrum der regulativen sozialen Mechanismen bezogen ist, befindet es sich entsprechend den Wandlungen der ökonomischen, politischen und sozialen Strukturen der bürgerlichen Gesellschaft in beständiger Veränderung; einer Veränderung freilich, die ihre Grenzen im vorausgesetzten Lohnarbeitsverhältnis hat, sich immer nur als Veränderung desselben darstellt.

Für eine Theorie des Arbeitsrechts bedeutet dies zweierlei: einmal kann sie nur auf der Basis einer gesamtgesellschaftlichen Theorie entwickelt werden, die in der Lage ist, den Zusammenhang von OKonomie und Politik, von gesellschaftlicher Stellung im Reproduktionsprozeß und Bewußtsein, von Theorie und Praxis zu erklären. Zum anderen muß sie Auskunft geben können über das Verhältnis von den für eine Gesellschaft konstitutiven zu den veränderbaren Faktoren, d.h. über den Zusammenhang von gleichbleibenden Grundstrukturen bei einem gleichzeitig großen Maß von historischer Flexibilität.

* An der Diskussion des Aufsatzes, der in seinem letzten Teil auf Ausführungen von Ulrich Müdkenberger aufbaut, waren neben diesem vor allem Rainer Erd und Bruno Schoch beteiligt. 
Die Arbeitsrechtswissenschaft ist an beiden Erfordernissen gescheitert. Als Kind der bürgerlichen Rechtswissenschaft, welche in ihrer theoretisch-dogmatischen Fixiertheit auf den Rechtsstaat dem Dualismus von Staats- und Verfassungstheorie einerseits und ökonomischer Gesellschaftstheorie andererseits verhaftet blieb, konnte sie dem besondern Charakter des Arbeitsrechts nicht gerecht werden; so mußte sie sich durch dessen faktische Entwicklung immer wieder über die Falschheit ihrer Vereinseitigungen belehren lassen.

Als liberales Vermögensrecht, wie es selbst noch das BGB konzipiert, ließ es sich spätestens nach Anerkennung der Tariffähigkeit der Gewerkschaften, der betrieblichen Mitbestimmung und der Einführung der staatlichen Schlichtungskompetenz in Arbeitskämpfen nicht länger begreifen. Ebensowenig läßt es sich vom Bezugspunkt einer materialen Staats- oder Verfassungstheorie her bestimmen: Die scheinbar totalen konzeptionellen Unterschiede als völkisches Ordnungsrecht im Nationalsozialismus wie als sozialstaatliches Gemeinschafts- und Fürsorgerecht in der BRD unterschlagen die weiterexistierende Identität des Arbeitsrechts als Vermögensrecht des Austauschs von Lohnarbeit und Kapital.

Dies Phänomen charakterisiert gerade die Besonderheit des Arbeitsrechts: daß es quer steht einmal zur relativ ungebrochenen Kontinuität des Zivil-, Prozeß- und Strafrechts und ihrer juristisch-dogmatischen "Durchdringung " seit den großen Kodifikationen im 19. Jahrhundert, zum anderen zur scheinbar gänzlichen Diskontinuität der Verfassungsgeschichte und -theorie; indem es so zwischen den großen "Blöcken " rechtswissenschaftlicher Theoriebildung, dem Zivil- und dem Offentlichen Recht, hindurchfällt, fristet es bis heute an den Universitäten ein kaum bemerktes Schattendasein. Diese - auch staatlich geförderte ${ }^{1}$ - wissenschaftliche Abstinenz gegenüber dem Arbeitsrecht steht nicht nur in umgekehrtem Verhältnis zu seiner objektiven gesellschaftlichen Bedeutung für den Großteil der Bevölkerung, sondern auch zu den Anforderungen und Konsequenzen, die seine Bearbeitung für eine Theorie des Rechts in der bürgerlichen Gesellschaft zur Folge hätte. Diese müßte sich nämlich nicht nur einer gesellschaftlichen Theorie sozialer Entwicklung und Veränderung vergewissern, sondern einer Theorie, welche die Mechanismen gesellschaftlicher Veränderung aus der realen Existenz antagonistischer sozialer Klassen entfaltet. Das gegenstandskonstitutiv auf diesen Antagonismus bezogene Arbeitsrecht wird daher nicht nur wegen der vielbeschworenen "Praxisferne « der Rechtswissenschaft zum Problem, sondern vor allem deshalb, weil es gleichsam den "Sprengsatz « gegenüber dieser in liberalidealistischen Harmonievorstellungen verfangenen Wissenschaft bildet. ${ }^{2}$

(II) In jüngster Zeit wird von einigen Autoren ${ }^{3}$ der Versuch wieder aufgenommen ${ }^{4}$, das Arbeitsrecht im Zusammenhang einer materialistischen Gesellschaftstheorie zu interpretieren ${ }^{5}$.

1 In keiner Ausbildungsordnung ist bisher die Teilnahme an einer Übung im Arbeitsrecht zur zwingenden Voraussetzung für das erste juristische Staatsexamen gemacht worden; die Zahl der Professoren für Arbeitsrecht ist entsprechend gering (Beispiel aus Frankfurt: I Professor für Arbeitsrecht gegenüber z. B. allein 8 für Rechtsgeschichte).

2 K. Korsch hat diese - auf der Basis der Negation des Antagonismus von Lohnarbeit und Kapital notwendige - Unfähigkeit der bürgerlichen Rechtswissenschaft, die Besonderbeit des Arbeitsrechts und seiner Entwicklungen zu begreifen, ausführlich dargestellt in seiner Antrittsvorlesung v. 9. 5. 1923 *Jus belli ac pacis im Arbeitsrecht « (zuerst veröffentlicht in $\mathrm{KJ} \mathrm{2/1972}$ S. $142 \mathrm{ff}$.).

3 Zu nennen wären hier z. B. die jüngssten Arbeiten von W. Däubler, R. Geffken, R. Hoffmann.

4 Aus der Wemarer Zeit vgl, die arbeitsrechtlichen Studien von O. Kahn-Freund, K. Korsch,

E. Fraenkel und F. Neumann.

5 Erste Ansätze, arbeitsrechtliche Entwidklungen bzw. Phänomene im Rahmen mederner sozial- 
Bislang beschränkten sich diese Untersuchungen überwiegend auf eine ideologiekritische Analyse herrschender arbeitsrechtlicher "Theorien « und der ihnen zugrundeliegenden gesellschaftlichen Ordnungskonzeptionen, wie sie in der juristischen Literatur, der Rechtsprechung und in Gesetzen gang und gäbe sind. Dabei erscheinen diese ideologiekritischen Arbciten jedoch gegenüber dem Anspruch einer materialistischen Ideologiekritik insofern verkürzt, als sie sich regelmäßig darauf beschränken, die bekannten arbeitsrechtlichen Denkfiguren (freier Arbeitsvertrag, arbeitsrechtliches Gemeinschaftsverhältnis, Betriebsrisikolehre, Kampfparität etc.) vor dem Hintergrund der zentralen Kategorien der marxschen Politischen Okonomie (Warenform der Arbeitskraft, Doppelcharakter des Produktionsprozesses als Arbeits- und Verwertungsprozeß etc.) als gesellschaftliche Rechtfertigungslehren im Interesse der Aufrechterhaltung der kapitalistischen Produktionsverhältnisse zu entschleiern ${ }^{6}$. Gewiß sind solche Untersuchungen notwendig, schon um deutlich zu machen, wie sich ohnedies faktisch herrschende Partialinteressen in den Nebelgebilden bürgerlicher Ideologie zu Allgemeininteressen aufspreizen und in welchem Ausmaß sowie mit welchen Konsequenzen sie auf den verschiedenen Gebieten der arbeitsrechtlichen Rechtsprechung und Gesetzgebung Berücksichtigung finden. ${ }^{7}$

wissenschaftlicher Theorien zu interpreticren, sind bislang vereinzelt geblieben und wurden von juristischer Seite so gut wie überhaupt nıcht zur Kenntnis genommen; vgl. z. B. die systemtheoretischen Untersuchungen über die Funktionsmechanismen des Tarifvertragssystems von $\mathrm{H}$. J. Weitbrecht, Effektivität und Legimıtät der Tarifautoncmıe, Berlin 1969 und von L. Unterseher, Tarifliche Schlichtung: ein ökonomısches Gerich.tsverfahren?, in: KJ 3/197 I S. $278 \mathrm{ff}$. Auf Darstellung und Kritik dieser Versuche, das Arbeitsrecht sozialwissenschaftlich zu fundieren, muß hier verzichtet werden, weil dies eıne eigenständige Untersuchung im Rahmen eıner grundsätzlicheren Auseinandersetzung mit der Systemtheorie erfordern würde; vgl. zur Studie von Weitbreche T. Blanke, Funktionswandel des Streiks im Spätkapitalismus, Ffm 1972 S. 82 ff. mit weiteren Hinwessen.

6 Zwar zitiert R. Geffken (Das Individualarbeitsrecht, in: Demokratie und Recht, Nr. 2/73 S. $107 \mathrm{ff}$.) elnyanzs die Formulierung aus der "Deutschen Ideologie « von K. Marx/F. Engels: »Die ihre materielle Produktion und ihren materiellen Verkehr entwickelnden Menschen ändern mit dieser ihter Wirklichkeit auch ihr Denken und die Produkte ihres Denkens «. Das darin ausgesprochene differenzierte Wechselverhältnis von tätiger Produktion und objektiv veränderter Wirklichkeıt zu ihren gedanklichen Ausdrücken und der von ihnen wiederum angeleiteten gesellschaftlichen Praxis ( Produkte ihres Denkens «) reduziert sich aber im Forrgang der Untersuchung alsbald in der Ubernahme der Definition von W. Hofmann (Wissenschaft und Ideologie, in: Universität, Ideologıe, Gesellschaft. Beiträge zur Wissenschaftssoziologie, Ffm $1968 \mathrm{~S}$. $54 \mathrm{ff}$.) wieder zu einem Ideologrebegriff, der sich von dem »landläufigen* (Hofmann, S. 56) nur dadurch unterscheidet, daß er den Interesse-Charakter des ideologischen Denkens näher bestimmt: Ideologie ist danach "gesellschaftliche Rechtfertigungslehre", "Ausdruck des überlegenen Teils der Geselischaft... und (muß) geglaubt werden, wenn nicht von ihren Trägern ..., so jedenfalls von den Ideologieunterworfenen« (Geffken, a. a. O. S. I i 1 nach Hofmann, S. 54,55). Von diesem verkürzten Ideologiebegriff her erklärt sich die Reduktion der Untersuchung auf die Analyse der Interessen des »überlegenen Teils der Gesellschaft «: „Das Monopolkapital ging sehr rasch dazu über, dieses Rechtsgebiet (das kollektive Arbeitsrecht, T. B.) - war es einmal anerkannt - seinen Interessen unterzuordnen* (Gefken, a. a. O. S. 110).

$7 \mathrm{Vgl}$. W. Däubler, Das soziale Ideal des BAG, vv. Manuskript, 1973; Däubler lege seiner Analyse der BAG-Rechtsprechung ein Raster vorab zusammengestellter Interessen der Arbeitnehmer einerseits, der Unternehmer andererseits zugrunde und beurteilt danach, welche Interessen sich in der Rechtsprechung vorrangig durchsetzen. Zum Schluß seiner sehr materialreichen und manche allfälligen Einschätzungen wie z. B. die, daß das Individualarbestsrecht eine $n$ Art Eldorado des Arbeitnehmerschutzes « (a. a. O. S. 160) sei, gründlich widerlegenden Studie resümiert er: „Versucht man, die hier dargestellten und ansatzweise kritisierten Entscheidungen insgesamt zu würdigen, so wird die eindeutige und durchgängige Wahrung des unternehmerischen Rentabilitätsinteresses... deutlich * (a. a. O. S. I 56 ).

Ahnlich reduziert bleibt der Anspruch einer ideologiekritischen Analyse bei $R$. Hoffmann (Rechtsfortschritt durch gewerkschaftliche Gegenmacht, Ffm 1968): $\mathrm{Da}$ also das Recht als Machtfrage letztlich das Ergebnis gesellschaftlicher Auseinandersetzungen 1st, wird der Inhalt der Rechtsordnung, grundsätzlich und im einzelnen stets mehr oder weniger, von jenen Vorstellungen und Ideologien geprägt, die den im gesellschaftlichen Zusammenhang dominierenden Interessen und Schichten adäquat sind. Insoweit werden die Partikularinteressen der vorherr- 
Damit ist jedoch erst ein Teil einer materialistischen ideologiekritischen Analyse geleistet. Die sehr viel schwieriger zu beantwortende, dafür aber theoretisch wie politisch-praktisch sehr viel folgenreichere Frage nach den gesellschaftlichen Voraussetzungen für die Entstehung und Durchsetzung der jeweiligen ideologischen Denkmuster bleibt überhaupt erst zu stellen, wenn man sich nicht mit der im Grunde ebenso ahistorischen wie im Rahmen der marxschen Kritik der Politischen OKkonomie fast schon trivialen Feststellung begnügen will, daß den bürgerlichen Ideologien sämtlich die Funktion der Legitimation der bestehenden sozialen Verhältnisse zukommt.

Bei dieser Aussage stehen zu bleiben ist zum einen theoretisch unfruchtbar: In der abstrakten Gegenüberstellung herrschender juristischer Vorstellungen und der zentralen Kategorien der marxschen Kapitalismuskritik erstarren diese zu einer dogmatisch fixierten Begriffsapparatur, welche die Historizität dieser Theorie selbst nicht mehr reflektiert und damit die Aufgabe einer Rekonstruktion materialistischer Theorie entsprechend den veränderten ökonomisch-sozialen Reproduktionsformen der bürgerlichen Gesellschaft aus dem Blick verliert.

Die Gefahr einer Immunisierung der Theorie gegenüber historischen Wandlungsprozessen unterläuft an entscheidender Stelle den spezifischen Anspruch der materialistischen Gesellschaftstheorie: Sich von den wirklichen Verhältnissen immer wieder belehren zu lassen und nicht zu einem »materialistischen « Pendant bürgerlich-idealistischer Modellutopien zu degenerieren. Die Einlösung dieses Programms weist nicht nur der empirischen Forschung, sondern gerade auch den bürgerlichen Gesellschaftstheorien und den durchweg vorwissenschaftlichen "Alltagstheorien« juristischer Provenienz einen sehr viel bedeutsameren Stellenwert zu, als er in den bloß interessenkritischen Untersuchungen zum Ausdruck kommt; sie sind nämlich nicht nur schlicht als falsche und entstellende Vorstellungen über gesellschaftliche Zusammenhänge zu begreifen - wie dies in Entsprechung zur frühbürgerlichen »Priestertrugsthese« der Aufklärung mit der Behauptung einer ideologisch total manipulierten Geselischaft, in der demnach die Dialektik von Sein und Bewußtsein außer Kraft gesetzt wäre, behauptet wird $^{8}$ - sondern als partiell durchaus richtige begriffliche Darstellungen historisch jeweils bestimmter und daher durchaus verschiedener Erscheinungsformen sozialer Reproduktionsmechanismen an der warenzirkulierenden »Oberfläche « der kapitalistischen Gesellschaft.

schenden Gesellschaftsschichten durch die staatliche Rechtssetzung als , Allgemeininteresses sanktioniert « (a. a. O. S. 96). Entsprechend dieser Gleichsetzung von Ideologiekritik und Interessenanalyse kommt das Problem der objektiven Gencsis der Ideologıen aus den sozıalen Verhältnissen, ihr Charakter als durchaus richtige Ausdrucksformen einer sich verkehrt darstellenden gesellschaftlichen Realität und damıt die Bedeutung von Ideologiekritik für die Rekonstruktion je spezifischer historischer Wirklichkeit zu kurz; deutlich wird dies an der Untersuchung von $R$. Hoffmann "Der Grundsatz der Parität und die Zulässigkeit der Aussperrung«, vv. Manuskript, 1973, wo er nach einer Zurückweisung des herrschenden Paritärsdenkens auf Grund der sozioökonomischen »Ubermacht* (a.a.O. S. 14) der Unternehmerkoalitionen und einer differenzierten Analyse des Funktionswandels der Aussperrung auf die Frage zu sprechen kommt, warum die Gewerkschaften sich dieser Entwicklung der Rechtsprechung nicht widersetzt haben (S. 66 ff.) und resumiert: „Derart eingebunden als >Ordnungsfaktoren w wurden die Gewerkschaften gegenüber der massiv aufgedrängten Kampfformel einer 'Sozialpartnerschaft und damit verbundenen Paritätsvorstellungen weithin wehrlos« (S. 67).

8 Auffällig ist hier die Parallelität - wenn auch mit anderen politischstrategischen Folgerungen zur "Kritischen Theorie insbesondere Herbert Marcuses (vgl. insbesondere: Der eindimensionale Mensch, Studien zur Ideologie der fortgeschrittenen Industriegesellschaft, Neuwied-Berlin x 967) und Theodor W. Adornos (vgl. den Einleitungsvortrag zum r6. deutschen Soziologentag in Ffm in: Spätkapitalismus oder Industriegesellschaft? 1969, S. I 2 ff.), nach der der Spätkapitalismus als ein totaler Entfremdungs- und Verdinglichungszusammenhang erscheint, der durch die Allgemeinheit gezielter Bewußtscinsmanipulation die subjektiven Voraussetzungen einer antikapitalistischen proletarischen Massenbewegung liquidiert hat. 
Indem eine ideologiekritische Analyse in diesem Sinn daran festhält, daß die untersuchten Denkmuster keineswegs manipulativ-beliebige sind, sondern bewußtscinsmäßige Verarbeitungen gesellschaftlicher Wirklichkeit, individueller wie kollektiver Erfahrungen, eröffnet sie sich erst selbst wieder den Zugang zur je spezifischen gesellschaftlichen Realität, deren Ausdruck die Ideologien sind. Zugleich wird die Notwendigkeit erkennbar, sie als historisch unterschiedliche und in ihrer Genesis zu unterscheidende zu erklären, ihre Gemeinsamkeiten und Differenzen, Ungleichzeitigkeiten und Entsprechungen mit den zugrundeliegenden gesellschaftlichen Verhältnissen zu interpretieren ${ }^{9}$.

Eine materialistische Interpretation arbeitsrechtlicher Ideologien die sich auf eine interessenkritische Analyse beschränkt, greift aber nicht nur in theoretischer, sondern auch in politischer Hinsicht zu kurz. Für die Erarbeitung einer Aufklärungsstrategie, einer politischen Pädagogik, genügt nämlich das Wissen, daß sich in bestimmten Denkmustern soziale Befriedungsversuche des Kapitals verbergen, noch keineswegs ${ }^{10}$; vielmehr ist hierfür eine differenzierte Untersuchung der gesellschaftichen Entstehungsbedingungen solcher Ideologien erforderlich, der Gruppen und sozialen Klassen, die unter spezifischen sozialen Bedingungen derartiger Vorstellungen hervorbringen bzw. aufnehmen, sei es weil sie der Wahrnehmung gesellschaftlicher Erscheinungen aus ihrer Lage am ehesten entsprechen, sei es weil in ihnen - in welch' verkehrter Form auch immer - Momente kollektiver gesellschaftlicher Wunschvorstellungen oder allgemeiner, je individuell verdrängter Ängste eingehen; weiter müssen die gesellschaftlichen Rahmenbedingungen, unter denen bestimmte Ideologien sich als herrschende durchsetzen können sowie die Bedingungen, unter denen sie obsolet werden und andere an ihre Stelle treten, bestimmt werden.

Erst die Summe all dieser politökonomischen, soziologischen und sozialpsychologischen Bestimmungen ergibt ein annähernd präzises Bild von der gesellschaftlichen Relevanz bestimmter Ideologien und ermöglicht eine genauere Angabe der objektiven wie subjektiven Bedingungen, unter denen es möglich ist, ihren Verblendungszusammenhang zu durchschauen und damit politisch folgenreicher

9 Von dieser Frage der Bedeutung von Ideologien für die theoretische Rekonstruktion der bestimmten gesellschaflichen Realität, deren Ausdruck sie sind, ist die Frage zu trennen, ob die materialistische Gesellschaftstheorie, wie sie bei Marx ansatzweise ausgearbeitet vorliegt, nicht ihrerseıts in der Weise des kritisch-analytischen "Durchgangs\& durch die neueren Ansätze bürgerlicher gesamtgesel]schaftlicher Theorien vorab kategorial zu rekonstruieren wäre; diese Frage wird zumeist mit Hinweis auf von Marx getroffene Unterscheidung zwischen der für die Entwicklung seıner Kategorien der Politisehen Okonomie konstıtutiven *klassischen « bürgerlichen Okonomie und der bloßen Legitimationszwecken dienenden, daher theoretisch irrelevanten "Vulgärökonomie" verneint: Die gesamte "nachklassische »Okonomie - das gleiche gilt für Philosophie, Gesellschafts- und Wissenschaftstheorie - wird als bloße Verfallenserscheinung des bürgerlichen Denkens begriffen, welches einst in seiner »heroischen", historisch progressiven Phase als aufsteigende Klasse noch um die begriffliche Rekonstruktion gesellschaftlicher Totalität bemüht war, mit dem Verlust der gattungsspezifisch emanzipatıven Rolle der Bourgeoisie jedoch endgültıg seine aufklärerische Dimension verloren hat. Mir scheint, daß diese Frage nach dem »begriffskonstitutiven Status « der bürgerlichen Theorie für die Rekonstruktion materialistischer Gesellschaftstheorie sich mit dieser Erklärung weniger denn je begnügen kann: Noch immer beginnen die Desiderate materialistischer Gesellschaftstheorie ziemlich genau an den Problemen, die von Marx nicht mehr selbst ausgearbeitet wurden (z. B. Staats- und Rechtstheorie, Klassentheorie, Logik etc.).

10 Allzu leicht verfällt diese Form der Ideologiekritik in die Illusion, die jeweilige gesellschaftliche Realität immer schon als begriffen vorauszusetzen: "Die IIdeologies der Gewerkschaften aber muß die Waffe der wissenschaftlich exacten ökonomischen Wahrheit sein! « (Geffken, a. a. O. S. 123); entsprechend formelhaft postulativ bleiben die praktischen Konsequenzen einer solchen Untersuchung: »Entschiedener Kampf gegen die Verbreitung gefährlicher Begriffe wie »Personengemeinschaft * und die daraus abgeleiteten Kategorien, wie Treuepflicht, "Gehorsamspflicht * usw., Reduzierung des Arbeitsvertrages auf einen schuldrechtichen Austauschvertrag zweier ungleicher Parteien, durch ökonomische (nicht juristische) Begründung (Geffken, a. a. O. S. I23). 
»soziologische Aufklärung " zu initiieren: Dies ist das praktische Interesse an materialistischer Ideologiekritik.

(III) Eine erst noch zu konstituierende materialistische Theorie des Arbeitsrechts kaun sich indes nicht mit der Kritik arbeitsrechtlicher Ideologien begnïgen - auch wenn der Interpretationshintergrund so weit aufgefaltet ist, wie dies hier für erforderlich gehalten wird. Eine Theorie des Arbeitsrechts muß sich darüberhinaus mit der gesellschaftichen Funktion einzelner kollektivvertraglicher, gerichtlicher wie legislativer Maßnahmen, der Frage nach den Bedingungen, unter denen eine bestimmte Entscheidung so und nicht anders ergeht, allgemeiner: nach der "Logik « arbeitsrechtlicher Entwicklung insgesamt, beschäftigen.

Hierfür ist es erforderlich, die zentralen Aktionsparameter, die auf die Veränderung des Arbeitsrechts wesentlichen Einfluß haben, zu analysieren. Das Arbeitsrecht wurde einleitend ökonomisch als Ausdruck der kapitalistischen Produktionsweise, soziologisch als Ausdruck des Verhältnisses zwischen den entscheidenden sozialen Klassen bestimmt. Es folgt daher - und dies unterscheidet das Arbeitsrecht von anderen Rechtsgebieten - in seiner Entwicklung unmittelbar einer doppelten, freilich miteinander zusammenhängenden Logik: Der Akkumulation des Kapitals und den mit ihrem jeweiligen Stand gesetzten Reproduktionsbedingungen einerseits, dem politisch-sozialen Kräfteverhältnis zwischen den Lohnabhängigen ("Proletariat «) und den Unternehmern (»Bourgeoisie") andererseits. Prägnanter: Der je spezifischen Relation von Okonomie und Klassenkampf.

In der gesellschaflichen Aktion erscheint primär stets das politische Kräfteverhältnis, konkretisiert in einzelnen Maßnahmen, für die Beeinflussung arbeitsrechtlicher Entwicklung, die Erkämpfung bestimmter Rechtspositionen, relevant. Die nähere Bestimmung dieses Kräfteverhältnisses erfordert die theoretische Untersuchung einer Vielzahl von Faktoren:

- Des allgemeinen ökonomischen Kräfteverhältnisses zwischen den Klassen, wie es sich im Verhältnis von Angebot und Nachfrage auf dem Arbeitsmarkt und dem Anteil der Lohnabhängigen an der erwerbstätigen Bevölkerung insgesamt darstellt (Probleme der »industriellen Reservearmee «, der Lohnhöhe, der sozialen Schichtung der Lohnabhängigen in Arbeiter, Angestellte, Beamte).

- Des gesellschaftlichen Bewußtseins und sozialen »Anspruchsverhaltens" der Klassen und ihrer Schichtungen.

- Der formellen und informellen Regeln für die Austragung ökonomischer und politischer Konflikte.

- Der politischen und ökonomischen Organisationen der sozialen Klassen (Parteien, Gewerkschaften, Unternehmerverbände, shop stewards etc.), ihrer Organisationsstrukturen, Traditionen, Ideologien und Strategien.

- Des politischen Kräfteverhältnisses selbst, wie es sich in der Dominanz bestimmter Fraktionen des Kapitals und der jeweils regierenden konservativen bzw. reformistischen Parteien konkretisiert.

In diese allgemeinen Momente, die bloß analytisch, nicht aber in ihrer Genesis und in ihrem je aktuellen Zusammenhang voneinander isoliert werden können, löst sich jedoch die Frage nach dem politischen Kräfteverhältnis zwischen Lohnarbeit und Kapital, wie es zum Verständnis der Durchsetzbarkeit bestimmter rechtlicher Positionen erforderlich ist, keineswegs auf. Vielmehr wird die Relevanz, die diesen Faktoren für die Möglichkeit der Veränderung der rechtlichen 
Lage der Arbeiterklasse zukommt, inhaltlich bestimmt und begrenzt durch den je spezifischen Stand der Akkumulation und die ihm enisprechenden Verwertungsbedürfnisse des Kapitals, wie auch umgekehrt Ausmaß und Intensität der Akkumulation des Kapitals seinerseits beeinflußt werden durch das Kräfteverhältnis und die Auseinandersetzungen zwischen den gesellschaftlichen Klassen. Indes erscheint es richtig, daß den für die Entwicklung des Arbeitsrechts entscheidenden Aktionsparametern Ökonomie und Klassenkampf keineswegs ein gleichwertiger Einfluß zukommt, sondern daß vielmehr eine für die Entwicklung des Kapitalismus auch insgesamt charakteristische strukturelle Dominanz des Faktors Okonomie existiert: Wenn der ökonomische und politische Kampf der Arbeiterklasse in den kapitalistischen Ländern der $\gg 1$. Stunde « bis heute zu keiner qualitativen Veränderung der Produktionsverhältnisse geführt hat, sondern langfristig immer nur zur Durchsetzung solcher "Korrekturen « des Kapitalverhältnisses, die - zumindest aus der Sicht ex post - zugleich auch im objektiven Interesse des freilich ideellen, sich erst als Folge des Zusammenschlusses der Einzelkapitalien im Klassenkampf herstellenden Gesamtkapitals lagen, so erfordert die Interpretation bereits erkämpfter Rechtspositionen eine vorgängige Reflexion auf die allgemeinen Bedingungen und Formen der Reproduktion des Kapitals auf der gegebenen historischen Stufe. Das gleiche gilt für die Frage nach den Möglichkeiten, auf die Entwicklung des Arbeitsrechts durch die Erkämpfung neuer Rechtspositionen Einfluß zu nehmen: Eine realistische Gewerkschaftsstrategie, die die Illusionen eines kontinuierlichen »Rechtsfortschritts e ebenso wie das gebannte Starren auf die »revolutionäre Situation « - beides Ausdrücke einer ausschließlichen und isolierten Betrachtung des Faktors »politisch-soziales Kräfteverhältnis « - vermeiden will, muß in das Zentrum ihrer Analyse das logische Subjekt der kapitalistischen Gesellschaft, das Kapital, stellen. Erst auf der Basis der Untersuchung seiner notwendig widersprïchlichen Bewegungsformen und ihrer gesellschaftlichen Auswirkungen in neuen Formen und Inhalten sozialer Auseinandersetzungen, die in ihrem spontanen Charakter oftmals - um nicht zu sagen regelmäßig - gerade nicht als gezielte Aktionen im Rahmen langfristiger Strategien angesehen werden können, lassen sich jeweils konkrete Perspektiven folgenreicher gesellschaftlicher Veränderung formulieren: Eine antikapitalistische Strategie, die weder hinter den aktuellen Stand kapitalistischer Entwicklung zurückfällt noch die spezifischen sozialen Erscheinungsformen des gesellschaftlichen Antagonismus verfehlt, sondern permanent theoretisch und praktisch an der Gesellschaft lernt.

Auch hierbei handelt es sich, ebenso wie bei den Úberlegungen zum Problem einer materialistischen Ideologiekritik, um ein theoretisches Forschungsprogramm, welches erst noch der Ausführung bedarf. Es ist daher gegenwärtig nicht möglich, eine ausgearbeitete Analyse etwa zur Erklärung des Arbeitsrechts in der BRD und seiner gegenwärtigen Entwicklungstendenzen vorzulegen, zumal dies ohne die notwendige Rekonstruktion materialistischer Theorie in den nicht empirischen, abstrakten Kategorien der marxschen Politischen Okonomie auf der hierfür erforderlichen Konkretionsstufe nicht zu leisten ist.

(IV) In diesem Dilemma erschien es uns sinnvoll, vorerst den umgekehrten Weg zu gehen: Mit der Untersuchung einzelner exemplarischer Auseinandersetzungen zwischen Lohnarbeit und Kapital in einigen westeuropäischen Ländern zu beginnen und daraus Probleme und Fragestellungen zu gewinnen, die die weitere Arbeit inhaltlich strukturieren könnten. 
r. Um den Bezugsrahmen deutlich zu machen, innerhalb dessen die dargestellten Konfliktverläufe vorläufig interpretierbar sind, sollen zunächst einige Phänomene des hier interessierenden Verhältnisses von Ókonomie und Klassenkampf thesenhaft skizziert werden, die in den betreffenden Ländern erst nach Anschluß der jeweiligen Rekonstruktionsphasen eine spezifisch gesellschaftiche Signifikanz erhalten haben.

a) Hervorstechendstes Merkmal der kapitalistischen Entwicklung in den letzten Jahren ist - auf der Ebene des Gesamtkapitals - die sprunghafte Zunahme ökonomischer Funktionen des Staates, wobei für die hier verfolgte Fragestellung den Versuchen bzw. Maßnahmen einer staatlichen Regulierung der Lohnhöhe besondere Relevanz zukommt; denn die Effektivität staatlicher Einkommenspolitik, deren Notwendigkeit für die Reproduktion des Kapitals heute freilich genauer abzuleiten wäre, erfordert eine weitgehende Begrenzung der gewerkschaftlichen Tarifautonomie (Einschränkung des Streikrechts, auch soweit es bloß ökonomisches Streikrecht ist).

b) Den Auseinandersetzungen um eine staatliche Einkommenspolitik (mit dem Ziel einer längerfristigen Senkung der Lohnquote) entspricht auf der Ebene des Einzelkapitals die - ebenfalls nach Abschluß der jeweiligen nationalen Rekonstruktionsphasen in historisch neuer Dimension durchgesetzte - rapide Beschleunigung der Arbeitsintensität und Arbeitsproduktivität.

In den daraus resultierenden objektiven Veränderungen, der »Objektivierung « der individuellen Leistung im Arbeitsprozeß durch die Verfahren der analytischen Arbeitsplatzbewertung (zunehmende Transparenz des Zusammenhangs von Arbeits- und Verwertungsprozeß), der fortschreitenden "Vergesellschaftung « von Ausbildung, wissenschaftlicher Forschung und teilweise sogar der Entwicklung neuer Technologien (verstärkte Abhängigkeit des Einzelkapitals von direkten und indirekten staatlichen Subventionen), machte sich der gesellschaftliche Charakter der Produktion selbst in der Natur der ergriffenen Maßnahmen geltend und geriet die private Aneignung und Verfügung über das gesellschaftlich produzierte Mehrprodukt in zunehmende Legitimationskrisen. Im Zuge dieser Entwicklung entstanden teils inhaltlich neue Konfliktzonen und Konfliktgruppen wie z. B. die Schüler-, Studenten- und Lehrlingsbewegung, teils veränderte Aktionsformen und -inhalte im traditionellen Konflikt zwischen Lohnarbeit und Kapital ("aktive Streiks«, Betriebsbesetzungen, Zunahme spontaner Streiks, Forderung nach gleichen Löhnen, nach Arbeiterkontrolle des Produktionsprozesses, "Arbeiterautonomie« etc.), teils verschärfte sich der Widerspruch zwischen den verschiedenen Kapitalfraktionen einerseits sowie zwischen Kapital und den an die Regierung gekommenen reformistischen Parteien andererseits (Verschärfung der Konkurrenz bei geringerer Profitmasse, Widerspruch zwischen zunehmender "Sozialisierung der Verluste «, der Notwendigkeit staatlicher Subventionierung der Produktion und privater Entscheidungsfreiheit der Unternehmer über Investition und Preise, Angst der Kapitalisten vor »kalter Sozialisierung « durch Steuer- und Kreditpolitik).

Diese zweite Ebene verweist auf die neue Form der Krisenhaftigkeit des Systems und die veränderte Militanz nicht nur der gewerkschaftlichen Basis, sondern auch und gerade solcher gesellschaftlicher Gruppen, die aus der Arbeiterbewegung bislang objektiv und subjektiv (etwa als Adressaten politischer Veränderungs- oder Revolutionsstrategien) ausgeschlossen waren.

c) Daraus resultiert das spezifische Spannungsfeld, in dem die Gewerkschaften in den letzten Jahren agieren: Den staatlichen Maßnahmen zu ihrer Integration in die aufgeblähte Apparatur politischer und ökonomischer Krisenvermeidungs- 
instanzen einerseits und der zu ihrer eigenen Selbsterhaltung vorausgesetzten

Notwendigkeit der Rezeption und Artikulation systemsprengender bzw. -transformierender Bedürfnisse ihrer organisatorischen Basis und der Erarbeitung von Strategien zu deren Durchsetzung.

2. Die nachfolgenden Darstellungen des Dockarbeiterstreiks 1972 in England, des »aktiven Streiks « bei LIP I 973 in Frankreich und der spontanen Streiks im Sommer und Herbst 1973 in der BRD beschreiben Konflikte, die auf der Basis ähnlich weit entwickelter kapitalistischer Gesellschaften entstanden sind. Auch sind die Faktoren, die diese Auseinandersezungen auslösten, keineswegs untypische oder neue: Umstrukturierungen ganzer Industriezweige auf Grund technischer Neuerungen (Container-Revolution in England), Reorganisation und Produktionsumstellungen in einzelnen Unternehmen auf Grund ökonomischer Konzentrationsprozesse und verschärften internationalen Wettbewerbs (Umstellung der Produktion bei LIP auf die "Wegwerfuhr ", Ausgliederung anderer Unternehmenszweige) und das Ansteigen der Lebenshaltungskosten trotz verschärfter Arbeitsintensität und -produktivität sind Ereignisse, die für die Entwicklung der kapitalistischen Gesellschaft charakteristisch sind und in den hier betrachteten Ländern beständig vor sich gehen.

Wenn sich an ihnen jedoch zu einem bestimmten Zeitpunkt soziale Auseinandersetzungen entzünden, die auf Grund ihrer Bedeutung gleichsam eine »konstitutionelle Dimension « (Generalstreikankündigungen in England und Frankreich, Appelle der Regierung und Spitzenverhandlungen von Gewerkschaften und Unternehmern in der BRDj erhalten, so wirft dies ein bezeichnendes Licht auf die veränderte und - bei allen oben beschriebenen Gemeinsamkeiten der aktuellen Strukturprobleme spätkapitalistischer Gesellschaften - im nationalen Rahmen höchst unterschiedliche Relevanz der einzelnen Momente kapitalistischer Entwicklung: Was in England, der BRD und Frankreich jeweils Anlaß für soziale Kämpfe ist, die sich fast zur »Systemkrise « auswachsen, bleibt in den jeweils anderen Ländern ein kaum bemerktes, alltägliches Ereignis ohne gravierende Folgen. Dies verweist auf die Unzulänglichkeit von Analysen, die auf der Ebene theoretisch abstrakter Generalisierungen aus dem Begriff des »Kapitals im Allgemeinen « stehen bleiben, für das Verständnis gesellschaftlicher Veränderungsprozesse im Verhältnis von Lohnarbeit und Kapital und damit die Interpretation arbeitsrechtlicher Entwicklungen. Die Legalität, die in allen Konflikten in Frage stand, war stets die gleiche: die bürgerliche. Die Rechtsnormen, die die Legalität jeweils im einzelnen bestimmen und um deren konkrete gesellschaftliche Geltung es jeweils direkt oder indirekt (auch) ging, waren jedoch gänzlich verschiedene: Das Arbeitsgesetz von I97 I in England, das Verbot "wilder « Streiks in der BRD, das Verbot des Diebstahls und der Hehlerei in Frankreich.

So stehen bei den Schilderungen der einzelnen Streikverläufe die logisch-kategorialen Widersprüche der kapitalistischen Gesellschaft nicht abstrakt zur Debatte, sondern ergeben sich gewissermaßen »induktiv « als Faktoren gesellschaftlicher Veränderung aus der Schilderung und Reflexion einer konkreten historischen Konfliktsituation und den Aktionen und Reaktionen der darin Beteiligten. Dabei erweist sich, daß der hier eingeschlagene Ausweg aus dem Dilemma einer fehlenden materialistischen "Realanalyse « der gegenwärtigen kapitalistischen Gesellschaft als Voraussetzung einer materialistischen Theorie des Arbeitsrechts nicht in eine theoretisch wie politisch-praktische Sackgasse mündet, sondern daß es sich vielmehr - wenn vorschnelle Verallgemeinerungen, kurzschlüssige Tendenzbestimmungen und euphorische Thriumphalismen vermieden werden - um 
notwendige Vorstudien handelt: Die begrifflich zu rekonstruierende gesellschaftliche Realität muß zunächst in ihrer konkret-historischen »Komplexität « unverkürzt dargestellt und analysiert sein, bevor die Aufgabe einer »Systematisierung des Chaos « weitergetrieben werden kann. Die Probleme, die sich hierbei stellen und die gegenüber einer abstrakten theoretischen Untersuchung notwendig veränderten Sichtweisen gesellschaftlicher Zusammenhänge sollen im folgenden diesen Aufsatz. abschließend - skizziert werden.

3. Jeden einzelnen der Streikverläufe könnte man analytisch in zahlreiche Einzelaspekte von jeweils großem theoretischen Gewicht auflösen. So könnte man etwa bezüglich des englischen Dockarbeiterstreiks von 1972 anhand der Container-Revolution in der Transportindustrie die Bedingungen und sozialen Auswirkungen des technologischen Fortschritts im Kapitalismus untersuchen und dabei den Zusammenhang von Dequalifizierung der Arbeitskraft auf Grund der Produktivkraftentwicklung und der Entfaltung von Klassenbewußtsein ausfindig machen. Untersuchenswert wären ferner die Verwertungszwänge im Reproduktionsprozeß des Kapitals, die den technologischen Wandel bald stimulieren, bald bremsen und in seiner konkreten Gestalt bestimmen. Daran schlösse sich die Untersuchung der im Zuge zunehmender Kapitalakkumulation wachsenden Verwertungsschwierigkeiten auf gesamtkapitalistischer Ebene an, die den Staat in neue Formen der Sicherung von Profitmargen hineinziehen, die den traditionellen Organisationen der Arbeiterklasse integrative Funktionen im gesamtgesellschaftlichen Reproduktionsprozeß aufnötigen, gerade dadurch aber wiederum neue Widersprüche in Gestalt unverfaßter Aktionen der Lohnabhängigen provozieren.

Eine derartige Isolierung von Einzelaspekten in Detailuntersuchungen würde jedoch unvermeidlich ein Moment von Abstraktion und Zerfaserung mit sich bringen, welches durch die Bündelung all dieser Faktoren in einer umreißbaren Konfliktsituation versuchsweise zurückgenommen werden soll zugunsten einer »konkreten Totalität«: der Vermittlung und Verschränkung der auf verschiedenen Ebenen wirkenden Faktoren, deren Zusammenhang erst einen historischen Prozeß ausmacht. Wenn Wissenschaft ihren Gegenstand Gesellschaft als in Veränderung begriffen interpretiert, dann stellt sich das ihr innewohnende Abstraktionsmoment ihrem - im Prinzip totalen - Deutungs- und Anleitungsanspruch gesellschaftlicher Praxis als Hindernis in den Weg. Dieses Problem, welches die erkenntnistheoretisch, aber bis heute nicht gesellschaftstheoretisch "überwundene « Kluft zwischen logisch-kategorialer und empirisch-historischer Analyse bezeichnet, macht sicl immer noch in dem prekären Widerspruch voluntaristisch-handlungsorientierender, streng genommen idealistischer Strategien der Arbeiterbewegung auf der Basis angeblich materialistischer Untersuchungen geltend.

a. Wenn in die Schilderungen der Konfliktverläufe weniger die logisch-kategorialen als die konkret-historischen Widersprïche eingehen, so ist dies nur cum grano salis zu verstehen. Nicht nur, weil eine solche Scheidung erkenntnistheoretisch nicht zu halten ist; sie würde auch dem praktischen Anspruch eines materialistischen Geschichtsverständnisses zuwiderlaufen, welches gerade die spezifischen historischen Umstände mit den gesellschaftsanalytischen Kategorien vermitteln will. Es geschieht daher nicht aus Not, sondern mit voller Absicht, daß kategoriale Bestimmungen in die Schilderungen eingehen und aus ihnen entwickelt werden. Diese Kategorien existieren nämlich nicht »an sich«, aus jedem 
historischen Kontext herausgelöst, sondern können aufgrund ihrer Allgemeinheit aus der jeweiligen Konfliktsituation abgehoben und in vergleichbaren Situationen wieder aktualisiert werden: Sie können erfahren und erlernt werden, um auch an anderen gesellschaftlichen Prozessen ihre Erklärungs- und praktische Anleitungskraft zu beweisen. Dies macht sie zu einem wichtigen Gegenstand politischer Bildung. Vom Grad der Ausbildung politischen Bewußtseins und historischer Sensibilität hängt es nämlich $a b$, inwieweit den Akteuren in gesellschaftlichen Auseinandersetzungen die Vermittlung der je spezifischen Konfliktbedingungen mit den allgemeinen gesellschaftstheoretischen Kategorien in ihrer unmittelbaren Praxis gelingt.

Die einzelnen Streikschilderungen sind Resultat von und Beitrag zu politischen Bildungsprozessen: Resultat, indem sie die Lernprozesse in der gesellschaftlichen Praxis der Arbeiter wiedergeben und auf den Begriff zu bringen suchen; Beitrag, indem sie - vermittels der daraus entwickelten Kategorien - diese gesellschaftliche Praxis verfügbar machen für die aktuellen oder potentiellen Akteure in anderen Situationen, die, obwohl sie konkret immer unterschiedlich aussehen werden, sich gleichwohl aus den gleichen kategorialen Bestimmungen begreifen lassen.

b. Was zu der Vermittlung der empirisch-historischen mit der logisch-kategorialen Ebene gesagt wurde, steht in engem Zusammenhang mit dem, was in der Politischen Bildung neuerer Zeit als »exemplarisches Prinzip« bezeichnet wird. Dieser Begriff ist häufig Mißverständnissen ausgesetzt: In dem Maße, wie das exemplarische Prinzip nur noch als pädagogisches Vermittlungsinstrument verstanden und nicht mehr als Inhalte strukturierendes Prinzip begriffen wird, verliert es die kritische Dimension. Selbst dort, wo die praktische Dimension, die Motivation und die gesellschaftlichen Bedingungen für soziales Handeln immerwieder angesprochen werden, wie in der grundlegenden Schrift Oskar Negt's zur Arbeiterbildung, ist das exemplarische Prinzip vorwiegend auf der kognitivn Ebene entfaltet. Gegenüber der Vermittlung erlebbarer Einzelheit und gesellschaftlicher Totalität auf der Ebene des soziologischen Denkvermögens treten die Bedingungen gesellschaftlicher Praxis - obwohl als Anspruch formuliert zurück.

Weiterführend könnte man das exemplarische Prinzip als Kritik bloß kognitiver Lernprozesse formulieren. Gesellschaftliche Praxis hat auch mit Momenten zu tun, die sich dem rein kognitiven Zugriff entziehen. Solidarität, Kampferfahrung, Selbstbewußtsein, Zutraun, Freude über Erfolge sind entscheidende Elemente erfolgreicher politischer Bewegungen. Natürlich können diese Gefühle nicht die objektive Möglichkeit gesellschaftlicher Veränderung hervorbringen, aber sie müssen zu ihr hinzutreten, sonst findet die Veränderung nicht statt. Diese Gefühle sind keine theoretisch unergründbaren, invariablen Mächte: sie entwickeln sich im Zusammenhang mit bestimmten Sozialisationen, nehmen Erfahrungen in sich auf und bleiben veränderbar. An der Ausbildung und Bedeutung, die solche Gefühle für einen konkreten Konfliktverlauf haben, sind weiterhin kontingente, "zufällige Faktoren entscheidend: Personen, Situationen, Symbole, die zum Sinnbild ganzer Erlebnis- und Interpretationszusammenhänge haben werden können.

Indem in den Streikschilderungen einige dieser Faktoren deutlich werden, zeigt sich erneut die Notwendigkeit konkreter Rekonstruktionen gesellschaftlicher Veränderungsprozesse: Sie vermeidet die Schwäche einer Theorie, deren Abstraktionen immer noch notwendig bei der Herausarbeitung der Kategorien die 
Bedingungen ausblendet, die für die Handlungsmotivation - und damit die gesellschaftliche Praxis - unabdingbar sind.

c. Kontingente Faktoren, "Zufälle« wirken sich in der Geschichte aus und entziehen sich gleichwohl dem abstrahierenden Zugriff. Mit dieser Feststellung muß man nicht notwendig der Inkonsistenz und personalistischen Verkürzung des bürgerlichen Geschichtsverständnisses aufsitzen, das Geschichte notdürftig aus unvorhergesehenen Ereignissen und großen Persönlichkeiten ex post zusammenflickt. Die kontingenten Faktoren sind selbst wieder gesellschaftlich vermittelt und der Analyse zugänglich. Aber der abstrahierenden Wissenschaft ist die Präsenz sämtlicher Erklärungszusammenhänge in einer historischen Veränderungssituation nicht möglich. Gesellschaftliche Praxis tritt so an die Stelle der Wissenschaft im historischen Augenblick, in dem Veränderung möglich und notwendig ist; Spontaneität ist das Organ und Aktionspotential, das durch die Bündelung der geschichtswirksamen Faktoren hindurch die historische Notwendigkeit im je spezifischen und konkreten Moment - im doppelten Sinn - "wahrnimmt «. 